\title{
Perturbation Growth in Baroclinic Waves
}

\author{
Martha R. Stevens and Gregory J. Hakim \\ University of Washington, Seattle, Washington
}

(Manuscript received 14 July 2004, in final form 12 January 2005)

\begin{abstract}
Floquet theory is applied to the stability of time-periodic, nonparallel shear flows consisting of a baroclinic jet plus a neutral wave. This configuration is chosen as an idealized representation of baroclinic waves in a storm track, and the stability analysis may be helpful for understanding generic properties of the growth of forecast errors in such regions. Two useful attributes of Floquet theory relevant to this problem are that the period-average mode growth rate is norm independent, and the $t \rightarrow \infty$ stability limit is determined by the stability over one period. Exponentially growing Floquet modes are found for arbitrarily small departures from parallel flows.

Approximately $70 \%$ of Floquet-mode growth in energy is due to barotropic conversion, with the remainder due to zonal heat flux. Floquet-mode growth rates increase linearly with neutral wave amplitude (i.e., the "waviness" of the jet) and also increase with neutral wave wavelength. Growth rates for meridionally localized jets are approximately $40 \%$ smaller than for comparable cases with linear vertical shear (the Eady jet). Singular vectors for these flows converge to the leading Floquet mode over one basic-state period, and the leading instantaneous optimal mode closely resembles the leading Floquet mode.

Initial-value problems demonstrate that the periodic basic states are absolutely unstable, with Floquet modes spreading faster than the basic-state flow both upstream and downstream of an initially localized disturbance. This behavior dominates the convective instability of parallel-flow jets when the neutral baroclinic wave amplitude exceeds a threshold value of about 8-10 K. This result suggests that forecast errors in a storm track may spread faster, and affect upstream locations, for sufficiently wavy jets.
\end{abstract}

\section{Introduction}

Stability analyses have been applied to idealized shear flows representative of midlatitude jet streams in an attempt to better understand the development of extratropical cyclones and the growth of forecast errors. Although idealized time-independent flows have proven very useful for the cyclogenesis problem, they lack the temporal evolution typical of the forecast-error problem. Idealized periodic shear flows are a logical generalization that have intermediate complexity between time-independent and general aperiodic flows. Here we study the stability of a periodic basic state consisting of a jet plus a neutral mode of the jet, which we take as an idealization of a storm track.

The simplest shear flows are time independent and vary in only one direction-for example, the linear ver-

Corresponding author address: Gregory J. Hakim, Department of Atmospheric Sciences, University of Washington, Box 351640, Seattle, WA 98195-1640.

E-mail: hakim@atmos.washington.edu tical shear of the Eady (1949) jet. The Eady stability problem can be solved analytically by linearizing the governing equations and seeking exponentially growing normal modes. A similar stability analysis applies for meridionally localized jets (e.g., Hoskins and West 1979); however, although more realistic, these parallel flows still lack the time dependence of real jets.

Linear stability analysis is generalized to arbitrary unsteady flows through Lyapunov vector calculations (e.g., Legras and Vautard 1995). The leading exponentially growing Lyapunov vector dominates in the $t \rightarrow \infty$ limit and is norm-independent. However, it is often of interest to look at the stability of discrete time periods. Optimal modes (singular vectors) may be found for a chosen norm and time interval, and they typically grow faster than the leading Lyapunov vectors over discrete time periods (Farrell and Ioannou 1996). A disadvantage of optimal modes is their dependence on the chosen norm and time interval, both of which are arbitrary.

Periodic flows offer many of the complexities of fully time-dependent flows, but have the desirable property that the $t \rightarrow \infty$ stability limit is determined by the sta- 
bility over a single period. Moreover, the growth rates of unstable modes are norm-independent over a full period. The general theory for periodic flows derives from Floquet theory, which will be described in the following section. Although still idealized, the superposition of a baroclinic wave on a jet is a periodic flow that offers an approximation to the midlatitude storm tracks (e.g., Lim and Wallace 1991).

Stability analyses of periodic jet streams date back at least to Hart (1971), who found that the introduction of periodic time dependence to the Eady (1949) jet did not change the linear stability properties from the case of the time-independent jet. Duffy (1978) considered the stability of a basic state defined by the most unstable Eady mode in the absence of vertical shear. He found that this state supported unstable modes, which grow by both baroclinic and barotropic energy conversion. Samelson (2001) computed Floquet vectors for periodic, and nearly periodic, basic states for a simple model of wave-mean-flow interaction. Periodic states in this model were found to be stable, although weakly growing Floquet modes exist for slightly aperiodic limit cycles; these growing modes correspond to the unstable growth of the basic-state wave. This work was extended to a strongly nonlinear, aperiodic system, with similar results (Samelson and Wolfe 2003). Fantini and Davolio (2001) determined the leading mode for a neutral surface Eady wave superimposed on the Eady jet (linear vertical shear) by computing long integrations of a numerical model. For small-amplitude neutral waves they recovered the leading mode of the Eady model, but a new leading mode appeared when the neutral wave amplitude exceeded a threshold value. Here we build upon this work by showing that, among other things, these new modes exist for arbitrarily small neutral wave amplitude, and that the modes are robust to the introduction of meridional localization in the jet stream.

Aspects of Floquet theory relevant to this paper are summarized in section 2, and the numerical application of this theory is described in section 3. Results for the superposition of a neutral mode on the Eady jet and on localized jets are discussed in section 4. Section 5 is devoted to initial-value problems and the development of a localized disturbance. Section 6 provides a summary.

\section{Floquet theory}

The basic states considered here are periodic in time, suggesting the application of Floquet theory, which applies to systems of ordinary differential equations having periodic coefficients. The discrete version of the dynamical system is described by

$$
\frac{d \mathbf{x}}{d t}=\mathbf{L} \mathbf{x}
$$

where the $N \times N$ matrix $\mathbf{L}$ is a linear operator with period $t_{p}$, and $\mathbf{x}$ is the $N \times 1$ state vector of the system; the specific definitions of $\mathbf{L}$ and $\mathbf{x}$ are given in section 3 . A solution of (1) is

$$
\mathbf{x}(t)=\mathbf{M}\left(t, t_{0}\right) \mathbf{x}\left(t_{0}\right) .
$$

We shall refer to the $N \times N$ matrix $\mathbf{M}$, which evolves the state forward in time, as the propagator matrix. In particular, the solution after one period is

$$
\mathbf{x}\left(t_{p}\right)=\mathbf{M}\left(t_{p}, t_{0}\right) \mathbf{x}\left(t_{0}\right) .
$$

Because of the periodicity of $\mathbf{L}, \mathbf{M}\left(n t_{p}, t_{0}\right)=\left[\mathbf{M}\left(t_{p}, t_{0}\right)\right]^{n}$ where $n$ is any positive integer. Thus, the solution over an arbitrarily long time interval defined by $n$ periods is

$$
\mathbf{x}\left(n t_{p}\right)=\mathbf{M}\left(t_{p}, t_{0}\right)^{n} \mathbf{x}\left(t_{0}\right) .
$$

The Floquet-Lyapunov theorem states that the propagator matrix may be written as $\mathbf{P} e^{\mathbf{R} t}$, where $\mathbf{P}$ is periodic and $\mathbf{R}$ is a constant matrix (Yakubovich and Starzhinskii 1975). This proves that the system supports unbounded growth if there exists at least one eigenvalue of $\mathbf{R}$ having a positive real part. Therefore, an eigenanalysis of the propagator matrix gives the growth rates for one period,

$$
\sigma_{i}=\frac{1}{\left(t_{p}-t_{0}\right)} \ln \left(\lambda_{i}\right),
$$

where $\lambda_{i}$ represent the eigenvalues of the propagator matrix (also referred to as Floquet multipliers). The eigenvectors of $\mathbf{M}\left(t_{p}, t_{0}\right)$, which we call Floquet vectors, give the structures of the modes. Thus, an eigenanalysis of the propagator matrix for one period yields the stability for both that period and for $t \rightarrow \infty$.

Floquet vectors are equivalent to the Lyapunov vectors for this periodic flow and describe the asymptotic behavior of the system; however, they also describe the behavior for discrete time intervals (i.e., one period). Unlike singular vectors (optimal modes), which are also useful for assessing finite-time stability, Floquet vectors are independent of the norm over a period.

\section{Method}

To find the Floquet vectors and eigenvalues we need to first find the propagator matrix for the chosen periodic basic state. Typically, the stability of periodic flows is studied through an eigenfunction expansion that transforms the system to Mathieu's equation [e.g., Benjamin and Ursell (1954), as summarized in Drazin and Reid (1981, section 48.2)]. The stability of Mathieu's 
(a)

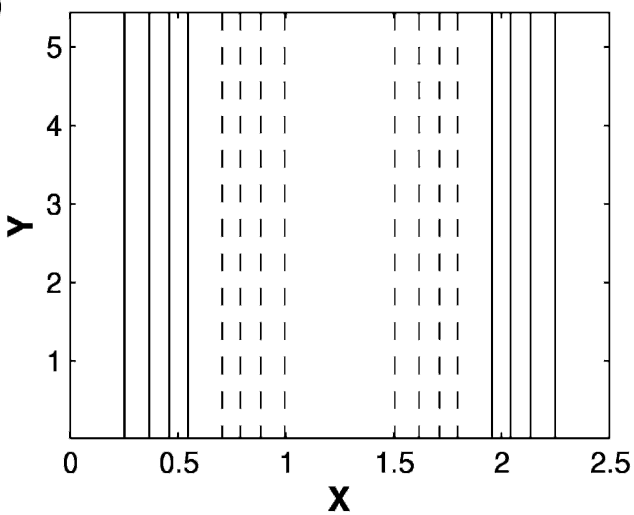

(c)

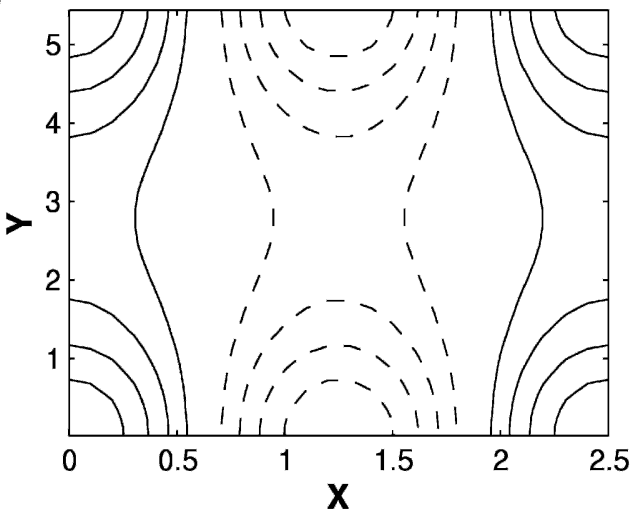

(b)

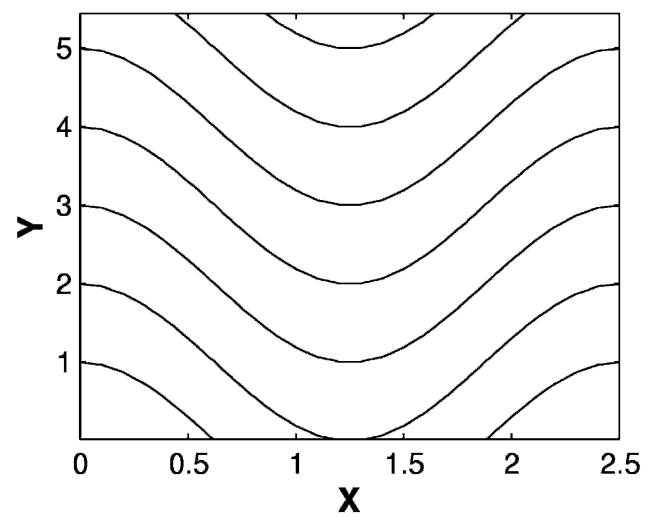

(d)

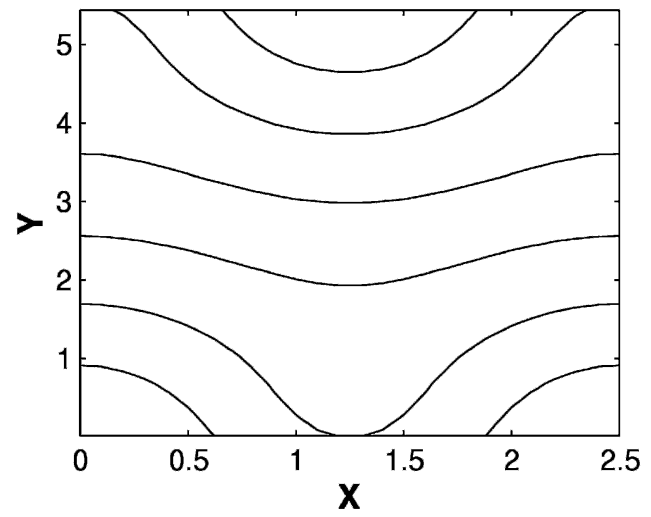

FIG. 1. Tropopause potential temperature fields for the (a), (b) $\mu=0$ and (c), (d) $\mu=0.2$ periodic basic states. (a) and (c) show the neutral wave every 0.2 units, and (b) and (d) show the combined jet and neutral wave field every 1 unit. Dashed lines represent negative values.

equation, which has been studied in great detail (e.g., Abramowitz and Stegun 1972, section 20.3), can be described as parametric resonance between frequencies of free and forced oscillations of the system. Unlike Benjamin and Ursell's study, we consider flows with spatial variations in three dimensions. Since it is not obvious how to transform these flows into the one-dimensional Mathieu equation, we resort to numerical methods. We consider now the basic states of the study, and the numerical methods for calculating the propagator matrix and the Floquet vectors.

\section{a. Basic state}

Our periodic basic states consist of a neutral mode superimposed on a time-independent jet. The simplest jet that we consider is the Eady jet, which has linear shear in the vertical and normal modes with zero potential vorticity in the interior of the domain. For $l=0$ (no mode structure in $y$ ), the superposition of a neutral mode and the basic-state jet is a finite-amplitude, nonlinear solution of the Eady model. For simplicity and numerical tractability, we limit both the basic states and solutions to zero potential vorticity so that the entire solution is given by the potential temperature at the surface and the tropopause. This common approximation is motivated both by observational evidence showing that potential vorticity gradients are concentrated at the tropopause (e.g., Hakim 2003) and by idealized investigations showing that flow stability properties do not depend sensitively on this choice (e.g., Harnik and Lindzen 1998; Song and Nakamura 2000). Modes of the Eady jet that have wavelengths shorter than a cutoff value are stable (e.g., Pedlosky 1987, section 7.7). Figure $1 \mathrm{~b}$ shows an example of a periodic Eady basic state, which is a sum of the Eady jet and a neutral Eady mode with a wavelength of $2500 \mathrm{~km}$. We shall refer to the stability analysis of this basic state as the Eady Floquet problem.

As a step toward more realistic flows, we consider the stability of basic states that contain meridionally localized jets. The Hoskins and West (1979, hereafter HW) jet adds structure in $y$ to linear shear:

$$
\bar{u}(y, z)=z-\frac{\mu}{2}\left[z+\frac{\sinh (l z)}{\sinh (l)} \cos (l y)\right],
$$


where $l$ is a constant (1.1358) and the parameter $\mu$ controls the concentration of the jet. (Note that $\mu=0$ recovers the Eady jet.) In this study, we consider jets with values of $\mu$ ranging from 0.0 to 0.3 , which is sufficient to assess the impact of meridional variation, but lacks the numerical difficulties encountered for more concentrated jets. ${ }^{1}$

\section{b. Numerical methods}

The neutral normal modes for the jet (6) were found by standard eigenanalysis. For $\mu=0$, normal modes are distinguished from the singular neutral modes by phase speeds that do not correspond to discrete model levels (e.g., Pedlosky 1987, section 7.7). It is more difficult to identify the neutral normal modes of jets with nonzero $\mu$ from phase speeds alone, so the following method was used: First, the eigenanalysis on the unstable side of the short-wave cutoff easily determines the normal modes by their complex eigenvalues. By decreasing the wavelength just below the short-wave cutoff, the desired neutral mode is found by correlating the spatial structure of the growing modes with neutral modes having similar phase speeds. This process may be extended to progressively shorter wavelengths.

To reduce computational time required for the stability analysis, a uniform barotropic wind is added to the basic state with the same magnitude and opposite sign as the phase speed of the neutral wave. Adding this barotropic wind makes the flow steady and translates the instability without changing the growth rates or structures of the modes. Now, since $\mathbf{L}$ is not a function of time, $\mathbf{M}\left(t_{p}, t_{0}\right)$ simplifies to

$$
\mathbf{M}\left(t_{p}, t_{0}\right)=e^{\mathbf{L}\left(t_{p}-t_{0}\right)}
$$

therefore, an eigenanalysis of $\mathbf{L}$ gives the Floquet modes, and the growth rates are the real part of the eigenvalues of $\mathbf{L}$. Unit impulse initial conditions in a subspace of a spectral model yield columns of $\mathbf{L}$, as described more completely below. Unit impulses are also used without adding the barotropic wind to compute $\mathbf{M}$ by integrating the model over one period of the basic state. The two methods give extremely similar results, and given this agreement, we use the first method because it is computationally faster.

The numerical model used here is a linear version of the quasi-geostrophic spectral model described in Hakim (2000), which is periodic in both $x$ and $y$, and has rigid horizontal boundaries for the surface and the

\footnotetext{
${ }^{1}$ Critical lines for the neutral mode enter into the domain at higher values of $\mu$, creating a kink in the potential temperature field that is difficult to resolve numerically.
}

tropopause. Dynamics are governed by conservation of potential vorticity over the interior of the domain. Assuming no dissipation and zero basic-state potential vorticity, the nondimensional governing equation is

$$
\frac{\partial q}{\partial t}=-\bar{u} \frac{\partial q}{\partial x}-\bar{v} \frac{\partial q}{\partial y}
$$

where $q$ is the perturbation potential vorticity and $\bar{u}$ and $\bar{v}$ are the basic-state zonal and meridional winds, respectively. Potential temperature is conserved on the boundaries $(z=0$ and $z=1)$ :

$$
\frac{\partial \theta}{\partial t}=-\bar{u} \frac{\partial \theta}{\partial x}-\bar{v} \frac{\partial \theta}{\partial y}-u_{g} \frac{\partial \bar{\theta}}{\partial x}-v_{g} \frac{\partial \bar{\theta}}{\partial y} .
$$

Basic-state quantities are marked with overbars and $u_{g}$, $v_{g}$, and $\theta$ represent perturbation zonal geostrophic wind, meridional geostrophic wind, and potential temperature, respectively. The perturbation quasi-geostrophic potential vorticity is defined as

$$
q=\frac{\partial^{2} \psi}{\partial x^{2}}+\frac{\partial^{2} \psi}{\partial y^{2}}+\frac{\partial^{2} \psi}{\partial z^{2}}=\frac{\partial v_{g}}{\partial x}-\frac{\partial u_{g}}{\partial y}+\frac{\partial \theta}{\partial z},
$$

where $\psi$ is the perturbation streamfunction. The model employs a fifth-order Adams-Bashforth time-differencing scheme.

We nondimensionalize as follows: the horizontal length scale $(L)$ is $1000 \mathrm{~km}$, the vertical length scale $(H)$ is $10 \mathrm{~km}$, the horizontal velocity scale $(U)$ is $30 \mathrm{~m} \mathrm{~s}^{-1}$, the time scale $(L / U)$ is $9.26 \mathrm{~h}$, and the potential temperature scale $\left[\theta_{0} f U L /(g H)\right.$, where $\theta_{0}$ is a constant value of potential temperature, $f$ is the constant Coriolis parameter, and $g$ is gravity] is $9.18 \mathrm{~K}$. Hereafter, values quoted without units are nondimensional, and dimensional values are placed in parentheses. The domain width is $5.5319(5531.9 \mathrm{~km})$ and the depth is 1 (10 $\mathrm{km}$ ). The length of the domain in $x$ is chosen to be the wavelength of the neutral wave that is added to the basic state.

A spectral subspace method is employed to insure that every degree of freedom in $\mathbf{L}$ ( or $\mathbf{M}$ ) is well resolved by the numerical model, which computes dealiased advection terms in gridpoint space. Specifically, the results shown here pertain to a model resolution of 32 by 32 horizontal wavenumbers (with 11 vertical levels), and unit impulses are applied to the subspace spanned by the leading eight horizontal wavenumbers in $x$ and $y$.

Three tests confirm the convergence of both the propagator matrix as well as the Floquet modes and growth rates. First, the numerically determined propagator matrix was benchmarked against the numerical model for a Gaussian initial condition; the solution de- 

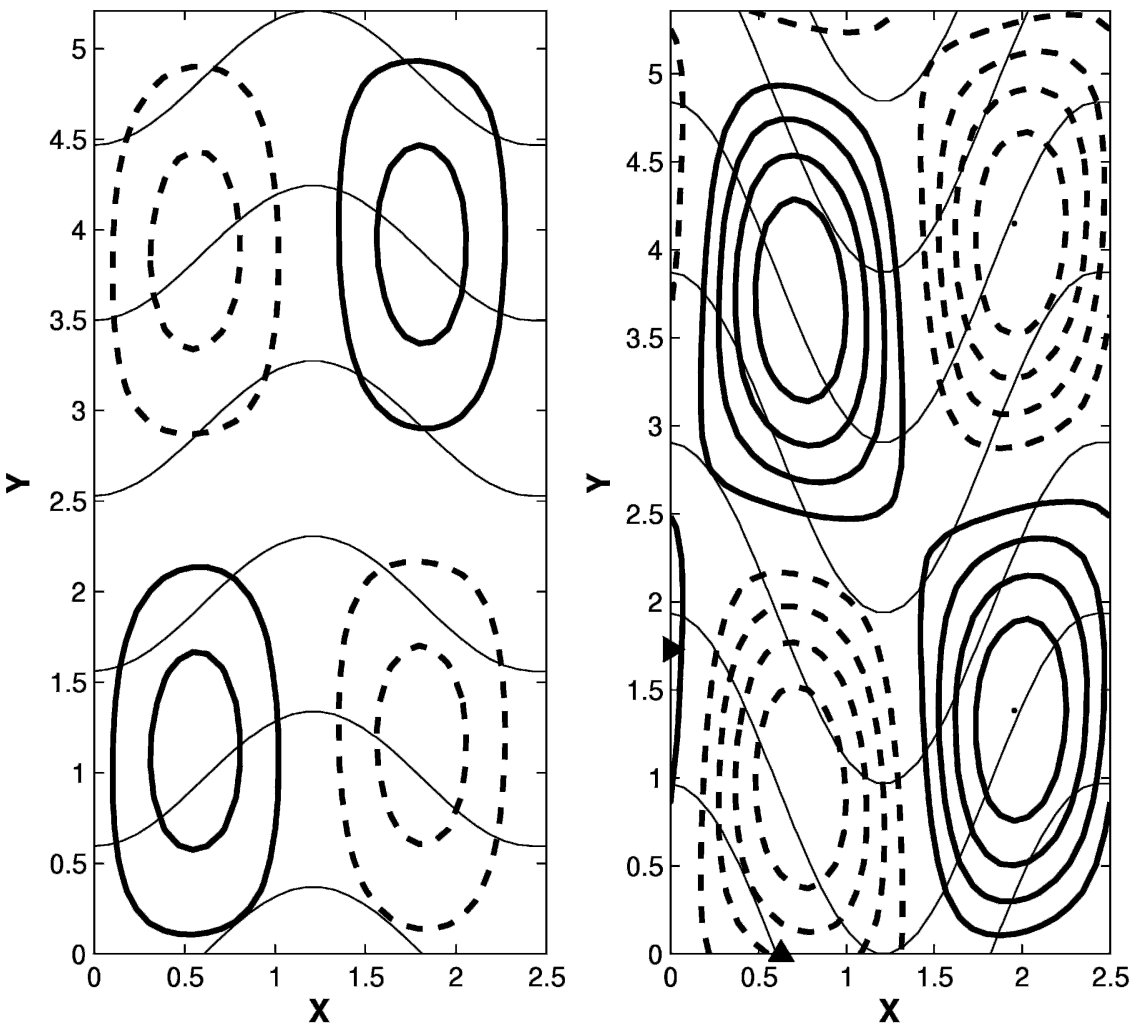

FIG. 2. The $\mu=0$ leading Floquet-mode potential temperature at the (left) surface and (right) tropopause for a neutral wave amplitude of 1 . Thin solid lines show basic-state potential temperature every 1 unit, and thick solid lines show the Floquet mode every 0.1 units at the surface and every 0.2 units at the tropopause; negative values are dashed. Triangles on the right panel mark the location of cross sections in Fig. 3.

termined by the product of the propagator matrix and the initial condition [i.e., (3)] compares well with the solution from the numerical model. Second, the robustness of the leading eigenvectors and eigenvalues was evaluated for several different model and impulse resolutions. Fixing the spectral impulse resolution at 8 by 8 and increasing the model resolution to 64 by 64 does not change the eigenvectors or eigenvalues (to order $10^{-5}$ ) for the $\mu=0.2$ Floquet problem. For $\mu=0$ the eigenvalues differ by $5.0 \times 10^{-3}$ with similar eigenvectors. Changing the impulse resolution from 8 by 8 to 16 by 16 also has very little impact on the results. The eigenvalues for the Eady Floquet problem change by $10^{-5}$ and the eigenvalues for the $\mu=0.2$ Floquet problem change by $1.3 \times 10^{-2}$, while the Floquet vectors do not noticeably differ. Increasing the vertical resolution from 11 levels to 41 levels changes the eigenvalues for the $\mu=0$ and $\mu=0.2$ Floquet problems by only $3 \%$. Finally, the Floquet modes determined from the eigenvectors of $\mathbf{L}$ were used as initial conditions in the numerical model and were found to be growing at the rate determined by the eigenvalues.

\section{Results}

\section{a. Eady problem}

Here we consider the stability of a neutral mode superimposed on the Eady $(\mu=0)$ jet (Fig. 1, top panels). The wavelength of the neutral mode is chosen to be 2.5 (2500 km, slightly on the stable side of the short-wave cutoff), which corresponds to a period of 4.371 ( $\approx 40.48$ h). Note that the classical unstable Eady modes are not supported in this domain, and recall that the tropopause-based mode is chosen to resemble baroclinic waves in a storm track.

After applying the methods discussed in the previous section to this problem, two growing modes were found through an eigenanalysis of the propagator matrix. The structure of the leading Floquet mode corresponding to a neutral wave amplitude of $1(9.18 \mathrm{~K})$ is shown in terms of perturbation potential temperature at both the surface and the tropopause in Fig. 2. The nondimensional growth rate associated with this mode is 0.35 , which compares with 0.31 for the most unstable mode of the classical Eady model. The leading mode is ap- 

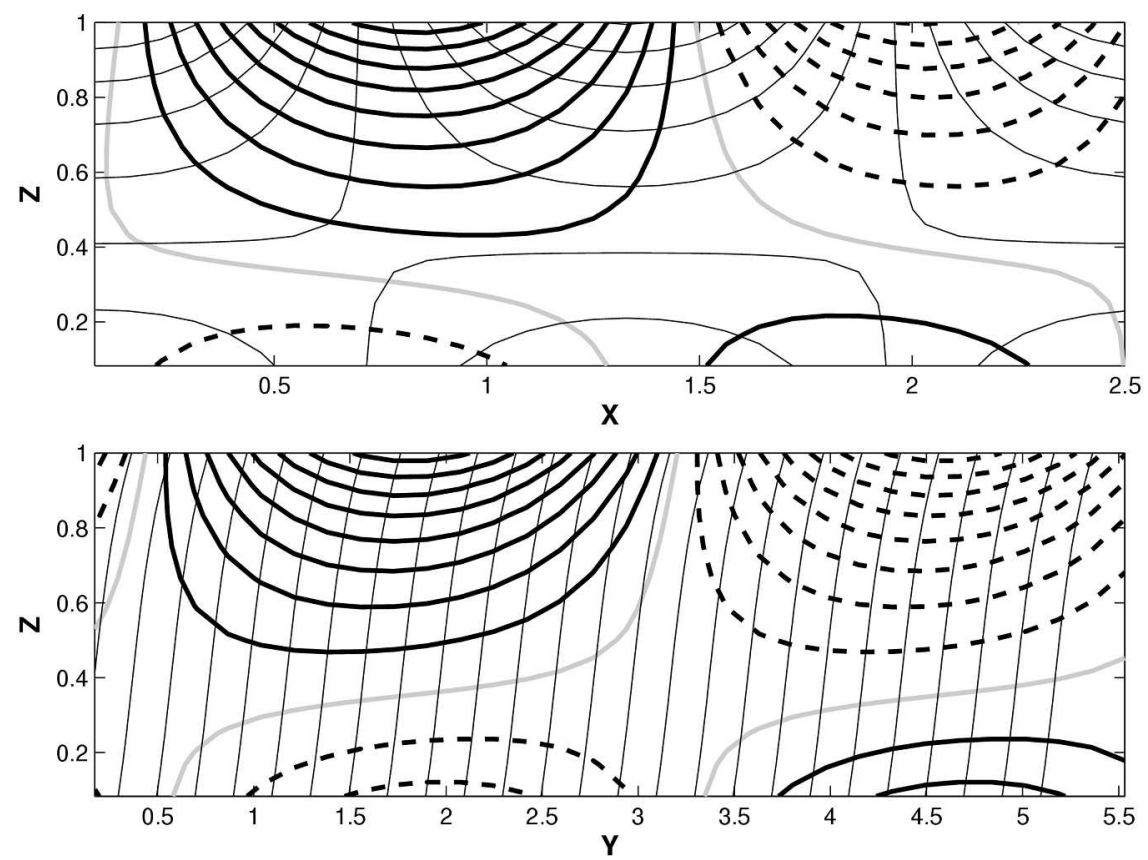

FIG. 3. Vertical cross sections of the $\mu=0$ leading Floquet mode for a neutral wave amplitude of 1 in the (top) $x-z$ plane at $y=1.73$ and (bottom) $y-z$ plane at $x=0.63$ (bottom). Thick lines show Floquet-mode potential temperature every 0.1 units with negative values dashed. The gray lines represent zero contours. Thin lines show the basic-state field every 0.2 units.

proximately a sine wave in both $x$ and $y$, with greater amplitude at the tropopause than at the surface. (Note the use of different contour intervals at the surface and the tropopause.)

Vertical cross sections of the leading mode in both $x$ and $y$ are given in Fig. 3. The thin solid lines represent the mean-state potential temperature, which reminds us that the neutral wave in the mean state has much greater amplitude at the tropopause. The Floquetmode potential temperature field exhibits maximum amplitude at the tropopause and an energetically unfavorable "westward" tilt in the $x-z$ plane for $y=$ $1.75(1750 \mathrm{~km})$. The mode potential temperature tilts "northward" in the $y-z$ plane (Fig. 3, lower panel).

These results correspond well with those of Fantini and Davolio (2001, hereafter FD), where a similar basic state consisting of a neutral surface Eady wave and an Eady jet was investigated. Based on long-term integrations of a numerical model, FD found two distinct classes of unstable modes depending on the amplitude of the neutral Eady wave. The first class corresponds to the familiar Eady-shear most unstable mode, and was found for small-amplitude neutral wave conditions. The second class was found when the amplitude of the neutral wave exceeded a threshold $(\sim 10 \mathrm{hPa})$. The growth rates of these modes increase linearly with Eady wave amplitude, and the phase speeds match that of the neutral wave. Our results are comparable to FD's second class of unstable modes. The structures of the leading Floquet mode and FD's second class of modes are very similar, and both modes have comparable growth rates that increase linearly with wave amplitude. FD's second class appears for a minimum wave amplitude of $10 \mathrm{hPa}$, whereas we find that the Floquet modes have growth rates greater than the Eady-shear most unstable mode at a wave amplitude of approximately $12 \mathrm{hPa}$. Regarding phase speed, we find that the Floquet mode moves slightly faster than the neutral mode, which will be examined further in section 5 .

The long integrations of FD only resolve the leading mode, and because their domain is large enough to support the most unstable Eady mode, their results suggest a threshold amplitude for growth of the Floquet modes. Here we find that the Floquet-mode growth rates have a linear relationship with neutral wave amplitude for all amplitudes (see Fig. 5). Furthermore, we find a second growing Floquet mode with a growth rate of 0.22 . The structure of this mode is similar to the leading mode, but is less symmetric in $x$ and $y$ (Fig. 4).

To quantify the growth of these modes, we consider the quasi-geostrophic disturbance energy equation 

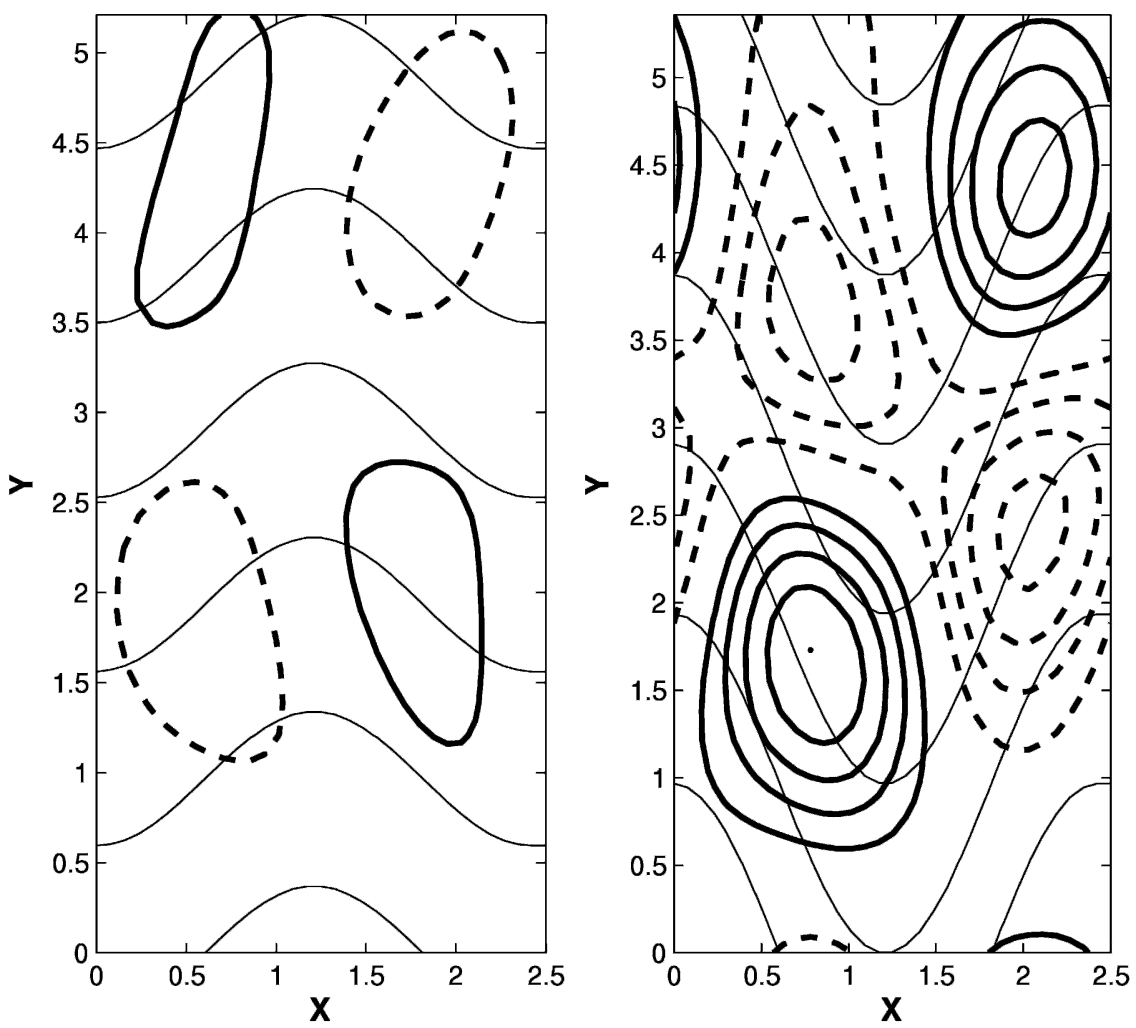

FIG. 4. As in Fig. 2, except for the second Floquet mode.

$$
\frac{d}{d t}\{E\}=\left\{\mathbf{E} \cdot \mathbf{D}_{m}\right\}-\left\{v_{g} \theta \frac{\partial \bar{\theta}}{\partial y}\right\}-\left\{u_{g} \theta \frac{\partial \bar{\theta}}{\partial x}\right\},
$$

where

$$
\begin{aligned}
& E=\frac{1}{2}\left[u_{g}^{2}+v_{g}^{2}+\theta^{2}\right], \\
& \mathbf{E}=\frac{1}{2}\left[\left(u_{g}^{2}-v_{g}^{2}\right),-u_{g} v_{g}\right],
\end{aligned}
$$

and

$$
\mathbf{D}_{m}=\left[\frac{\partial \bar{u}}{\partial x}-\frac{\partial \bar{v}}{\partial y}, \frac{\partial \bar{v}}{\partial x}+\frac{\partial \bar{u}}{\partial y}\right] .
$$

Equations (11)-(14) have been nondimensionalized according to the scaling parameters given in section $3 \mathrm{~b}$, and curly brackets denote a volume integral over the entire domain. Here $E$ represents the disturbance energy, and $\mathbf{E} \cdot \mathbf{D}_{\boldsymbol{m}}$ is the barotropic energy conversion term. The last two terms of (11) describe baroclinic energy conversion, the first being the meridional heat flux and second the zonal heat flux. Recall that the basic state includes a sum of the jet and a neutral wave. Hereafter references to growth rate apply to terms in (11) normalized by $\{E\}$.

For meridionally localized zonal jets, the barotropic conversion term is typically negative and reduces the growth rates of baroclinic waves (e.g., James 1987; Nakamura 1993). In contrast, for the leading Floquet mode, this term contributes approximately $70 \%$ of the energy growth rate (Fig. 5). The zonal heat flux, which reflects the mode tilt against vertical shear in the $y-z$ plane, contributes the remaining $30 \%$. The meridional heat flux, which is responsible for growth in the classical Eady model, contributes little; in fact, these values are slightly negative. These Floquet modes share similarities with the frontal wave instabilities described by Joly and Thorpe (1990), where both barotropic and baroclinic terms also contribute to growth. However, in Joly and Thorpe's study the baroclinic contribution was much greater than the barotropic contribution. Results for the second Floquet mode are similar, with barotropic conversion providing the greatest contribution to growth and the zonal heat flux also contributing positively. In summary, energy growth for the Floquet modes is due to both the horizontal shear and the temperature gradient in the $x$ direction.

Basic states with neutral wave amplitudes ranging from 0.5 to 3.0 ( 4.59 to $27.54 \mathrm{~K}$ ) show growth rates that increase linearly with wave amplitude and appear to go smoothly into the origin (Fig. 5); the individual terms 


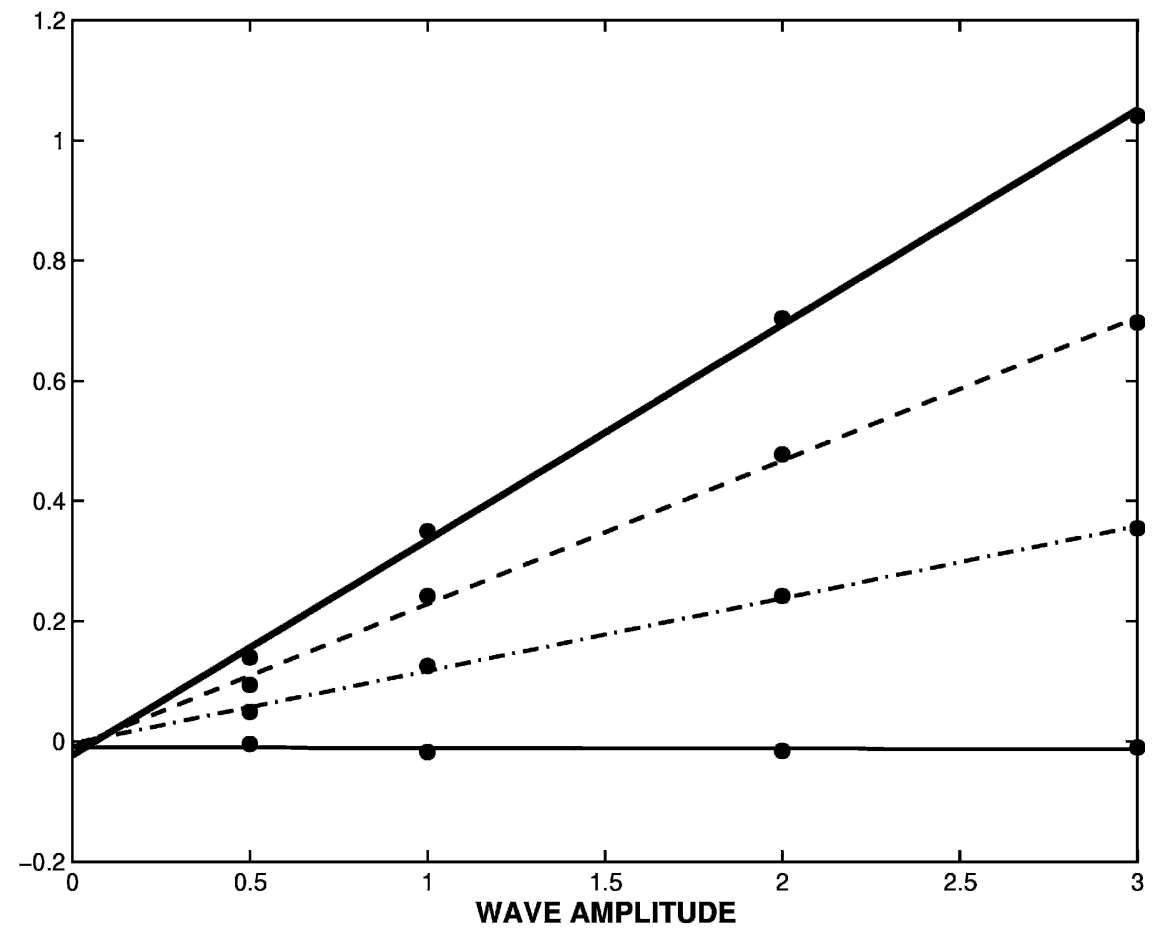

FIG. 5. Energetics analysis for the $\mu=0$ leading Floquet mode as a function of neutral wave amplitude: total energy growth rate (thick solid line), barotropic term (dashed line), zonal heat flux term (dashed-dotted line), and meridional heat flux term (thin solid line). Filled circles denote the computed values, and lines show a least squares linear fit to these values.

from the energetics equations also increase linearly with wave amplitude, as expected, since the horizontal shear and the $x$-direction temperature gradient are linear in neutral wave amplitude. As noted previously, for wave amplitudes greater than about $1(9.18 \mathrm{~K})$ the growth rate of the leading Floquet mode exceeds that of the Eady most unstable mode.

The growth rates and energetics results for a range of basic-state horizontal wavelengths are given in Table 1. Note that wavelengths greater than 2.6 are on the unstable side of the short-wave cutoff, and these waves are assigned a fixed amplitude of $1(9.18 \mathrm{~K})$ in order to maintain a periodic basic state. Growth rates increase with wavelength for the waves on the stable side of the short-wave cutoff (2.2-2.6), as both the barotropic term and the zonal heat flux term increase with wavelength. This result is somewhat counterintuitive, since an increase in wavelength for a fixed amplitude implies a widening of the shear zone and weaker horizontal shear. Recall that the energetics growth rate represents a volume integral, so the full three-dimensional domain must be considered. Shorter wavelength modes do indeed have stronger horizontal shear at the tropopause; however, the vertical extent of the Floquet modes (as well as the neutral waves) is smaller than for longer wavelengths. Thus when the barotropic term is integrated over the full domain, it has a smaller value for shallower waves with shorter wavelengths, despite the fact that they have larger horizontal shear at the tropo-

TABLE 1. Energy growth rate and individual contributions from the barotropic term $\left(\mathbf{E} \cdot \mathbf{D}_{\boldsymbol{m}}\right)$, meridional heat flux $\left[-v_{g} \theta(\partial \bar{\theta} / \partial y)\right]$, and zonal heat flux $\left[-u_{g} \theta(\partial \bar{\theta} / \partial x)\right]$ as a function of nondimensional wavelength for the periodic Eady jet. Wavelengths greater than 2.6 are on the unstable side of the short-wave cutoff; to render the basic-state periodic, these waves are assigned a fixed amplitude of one. The last row applies for a basic state with two neutral Eady waves.

\begin{tabular}{lcccc}
\hline \hline Wavelength & $\begin{array}{c}\text { Growth } \\
\text { rate }\end{array}$ & $\mathbf{E} \cdot \mathbf{D}_{\boldsymbol{m}}$ & $-\boldsymbol{v}_{g} \theta \frac{\partial \bar{\theta}}{\partial y}$ & $-u_{g} \theta \frac{\partial \bar{\theta}}{\partial x}$ \\
\hline 2.2 & 0.29 & 0.17 & 0.00 & 0.12 \\
2.3 & 0.32 & 0.20 & 0.00 & 0.12 \\
2.4 & 0.34 & 0.22 & 0.00 & 0.13 \\
2.5 & 0.35 & 0.25 & -0.02 & 0.13 \\
2.6 & 0.41 & 0.33 & -0.05 & 0.13 \\
2.7 & 0.54 & 0.35 & 0.05 & 0.14 \\
2.8 & 0.55 & 0.31 & 0.09 & 0.15 \\
3.0 & 0.55 & 0.24 & 0.14 & 0.17 \\
3.5 & 0.53 & 0.15 & 0.19 & 0.18 \\
3.9118 & 0.48 & 0.10 & 0.19 & 0.19 \\
2.5 & 0.11 & 0.07 & -0.01 & 0.05 \\
\hline
\end{tabular}


pause. Waves on the unstable side of the short-wave cutoff extend from the surface to the tropopause, and the barotropic term decreases as the wavelength increases due to the widening of the horizontal shear zone. The Floquet modes for these wavelengths lean against the vertical shear in both $x$ and $y$, so that the meridional and zonal heat flux terms are positive and increasing with wavelength. The decreasing barotropic terms and increasing baroclinic terms compete in the total energy growth rate, leading to a maximum value when the basic-state wavelength is approximately 3.0 $(3000 \mathrm{~km})$. For the Eady most unstable mode (wavelength 3.9118) with an amplitude of 1 (9.18 K), the leading Floquet mode has a growth rate approximately $60 \%$ larger than the Eady most unstable mode.

Basic states consisting of a growing wave superimposed on a jet are aperiodic, so Floquet theory does not strictly apply; right eigenvectors of the propagator matrix in this case have been called "finite time normal modes" (e.g., Molteni and Palmer 1993). However, for small growth rates, the flow is nearly periodic and we find results similar to the exactly periodic case summarized in Table 1. For example, the leading growing mode for a zonal wavelength of $2.7(2700 \mathrm{~km})$ with an initial amplitude of $1(9.18 \mathrm{~K})$ is very similar to the leading Floquet mode for the exactly periodic case (not shown). Most of the energy growth comes from barotropic conversion, and both baroclinic terms are positive throughout the period. Even for a strongly aperiodic case, such as a basic state consisting of the Eady most unstable mode with a wavelength of 3.9118 $(3911.8 \mathrm{~km})$ and an initial wave amplitude of $0.1(0.918$ $\mathrm{K})$, a growing mode exists that is similar to the periodic case (most unstable mode damped to neutrality). For this wave amplitude, the leading mode is the Eady most unstable mode and the second mode resembles the leading Floquet mode (not shown); this ordering reverses for larger-amplitude initial conditions.

The final case considered for the Eady jet involves the superposition of both the tropopause and surface neutral edge waves for a wavelength of $2.5(2500 \mathrm{~km})$. This configuration yields a basic state with a period of 34.8 (13.4 days) with periodic growth and decay in disturbance energy when the tropopause mode is upshear and downshear, respectively, from the surface mode (Rotunno and Fantini 1989). Relative to the case having only a tropopause mode, the leading Floquet vector for this transient state has a much lower growth rate of 0.11 . This lower growth rate is due primarily to a reduction in the barotropic conversion term, and also to a smaller contribution from the zonal heat-flux term (Table 1, last row). We conclude that large and rapidly changing vertical tilts of baroclinic waves reduces their Floquet instability.

\section{b. Concentrated jet results}

This section discusses results for basic states that vary in $y$. Results for $\mu=0.1,0.2$, and 0.3 are qualitatively similar; thus only the $\mu=0.2$ results will be discussed in detail. The basic-state potential temperature field is shown in Fig. 1d, which is composed of the $\mu=0.2$ jet and the tropopause-based neutral mode of that jet (Fig. 1c). Both the neutral mode and the jet have structure in $y$, with significant tropopause amplitude off the jet axis and surface amplitude along the jet axis. As $\mu$ increases from zero to 0.2 the tropopause-based neutral mode becomes concentrated off the jet axis and the surfacebased neutral mode concentrates at the center of the domain. Again, we consider a tropopause-based mode, which has a period of $5.022(\approx 46.50 \mathrm{~h})$.

The leading Floquet mode for an amplitude of 1 (9.18 $\mathrm{K})$ has a growth rate of 0.22 and vertical structure that peaks at the tropopause (Fig. 6). Structurally, the leading mode is similar to the $\mu=0$ leading Floquet mode except for greater amplitude off the jet axis. We note that there is a smooth transition from the $\mu=0$ leading Floquet mode to the $\mu=0.2$ leading Floquet mode and that the number of growing Floquet modes depends on neutral wave amplitude; two or three growing modes are found for most amplitudes.

Energetics results for these modes are very similar to the Eady Floquet case discussed earlier, with the growth rate and energetics terms increasing linearly with neutral wave amplitude (Fig. 7). The leading contributer to growth is the barotropic term, $\mathbf{E} \cdot \mathbf{D}_{\boldsymbol{m}}$, and the remaining energy growth comes from the zonal heat flux, $u_{g} \theta(\partial \bar{\theta} / \partial x)$. The meridional shear associated with the jet does act to reduce the contribution from barotropic growth; however, the reduction is small (only $0.9 \%$ when the neutral wave amplitude is one). Growth rates for several values of $\mu$ and different neutral wave amplitudes are given in Fig. 8. There is a linear increase in growth rate with amplitude for all of the values of $\mu$, and a decrease in growth rate for increasing $\mu$, with most of the reduction between $\mu=0$ and $\mu=0.1$.

The concentrated-jet Floquet results are similar to the Eady-jet Floquet results in several aspects. Both sets of results show a linear increase in growth rate with wave amplitude; approximately $70 \%$ of the energy growth is due to barotropic conversion; and no threshold amplitude for the instability. Furthermore, both leading Floquet modes have a sine-wave structure in $x$. The $\mu=0.2$ results differ from the Eady Floquet results by having somewhat lower growth rates and modes that are concentrated off the jet axis. 

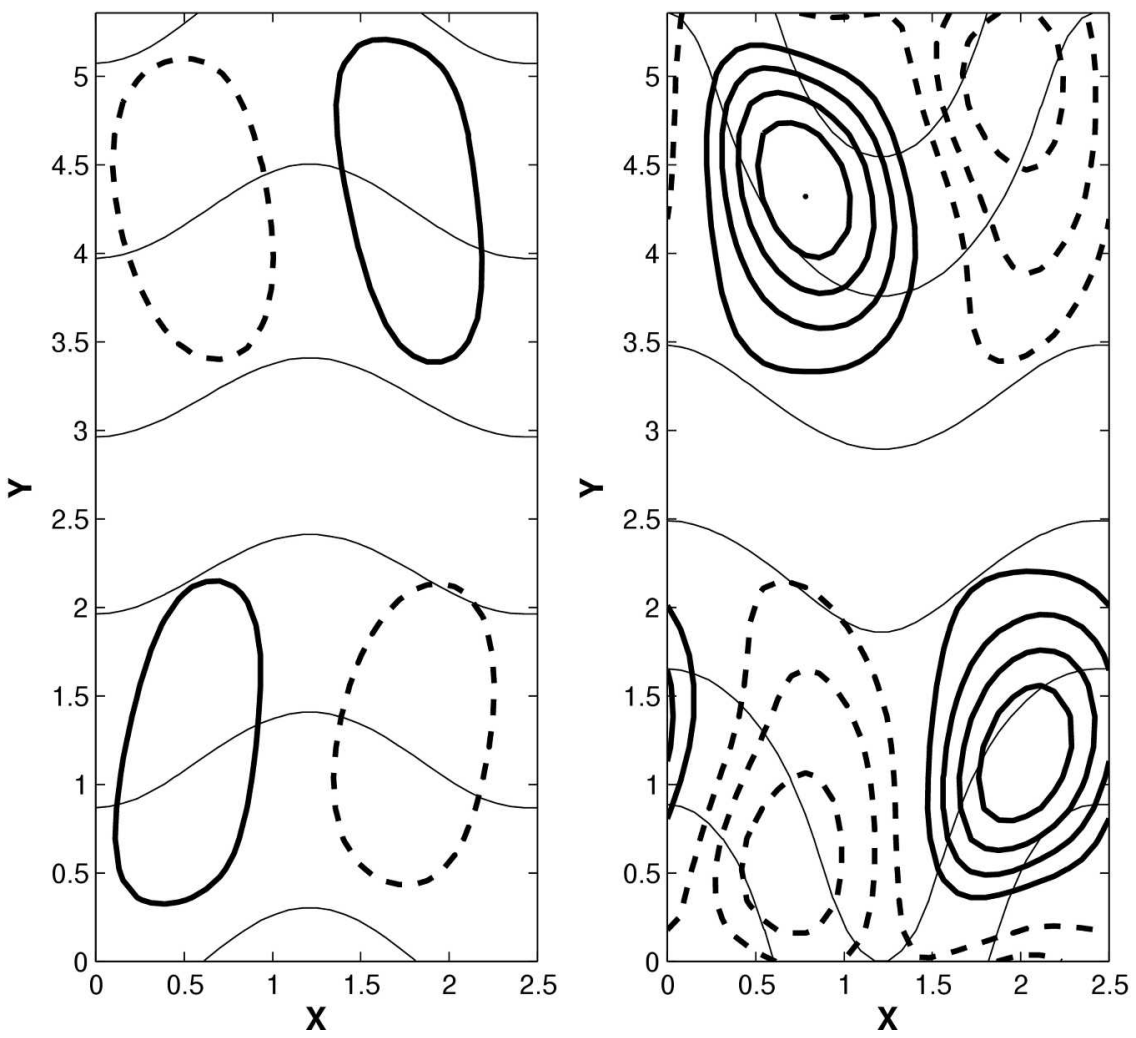

FIG. 6. As in Fig. 2, except for the leading Floquet mode of the $\mu=0.2$ basic state.

\section{c. Singular vectors}

As mentioned earlier, singular vectors provide another way to quantify stability over a discrete time period. The leading singular vector is the disturbance that amplifies most over a chosen time interval, as measured by a chosen norm (e.g., Farrell 1989). The amplification, $\alpha$, for the Euclidean norm is defined by

$$
\alpha^{2}=\frac{\langle\mathbf{x}(t), \mathbf{x}(t)\rangle}{\left\langle\mathbf{x}\left(t_{0}\right), \mathbf{x}\left(t_{0}\right)\right\rangle}=\frac{\left\langle\mathbf{M} \mathbf{x}\left(t_{0}\right), \mathbf{M} \mathbf{x}\left(t_{0}\right)\right\rangle}{\left\langle\mathbf{x}\left(t_{0}\right), \mathbf{x}\left(t_{0}\right)\right\rangle}=\frac{\mathbf{x}\left(t_{0}\right)^{\mathrm{H}} \mathbf{M}^{\mathrm{H}} \mathbf{M} \mathbf{x}\left(t_{0}\right)}{\mathbf{x}\left(t_{0}\right)^{\mathrm{H}} \mathbf{x}\left(t_{0}\right)},
$$

where ${ }^{\mathrm{H}}$ refers to the Hermitian transpose, and angle brackets denote the inner product. Since vector $\mathbf{x}$ contains the spectral coefficients of boundary potential temperature, this is a potential enstrophy norm. Eigenanalysis of $\mathbf{M}^{\mathrm{H}} \mathbf{M}$ gives singular vectors with amplification factors that are the square root of the eigenvalues.

The leading singular vector of the $\mu=0$ jet for one period of the $2.5(2500 \mathrm{~km})$ wavelength wave with an amplitude of 1 (9.18 K; 40.48-h period) has a periodaveraged exponential growth rate of 0.69 , which compares with 0.35 for the leading Floquet mode (Fig. 9, upper panels). The evolved singular vector is very similar to the leading Floquet mode (Fig. 9, lower panels); in fact, the Floquet-mode structure is evident as early as one time unit $(9.26 \mathrm{~h})$ into the evolution of the leading singular vector (not shown). The energy growth rate for this singular vector increases rapidly over the first time unit $(9.26 \mathrm{~h})$ and then converges to the growth rate of the leading Floquet vector (Fig. 10). Contributions to energy growth are similar to the Floquet mode, with the barotropic term responsible for approximately $70 \%$ of the growth during the entire period. The leading singular vectors at $t=0$ change significantly as a function of $\mu$ (not shown); however, we find that in all cases considered, the evolved singular vectors are very similar to the Floquet modes.

To assess the generality of this rapid convergence of singular vectors to Floquet modes, we consider two additional experiments. In the first experiment, singular vectors are determined for an aperiodic basic state defined by the most rapidly growing Eady mode. In the second experiment, instantaneous optimal modes are determined for a tropopause-based neutral mode with an amplitude of 2. These optimal modes represent the fastest growing perturbation at any instant in time; for our Euclidean norm, the leading optimal mode is de- 


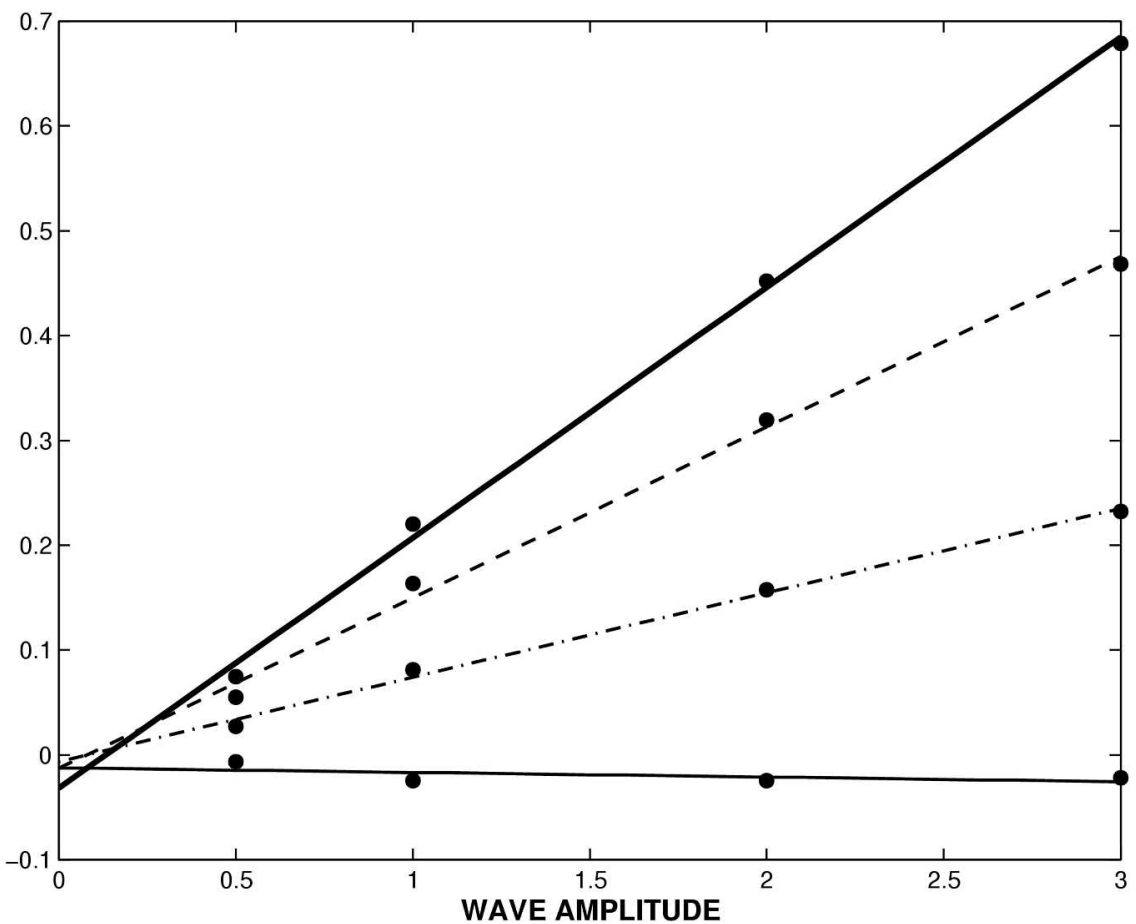

FIG. 7. As in Fig. 5, except for the $\mu=0.2$ leading Floquet mode.

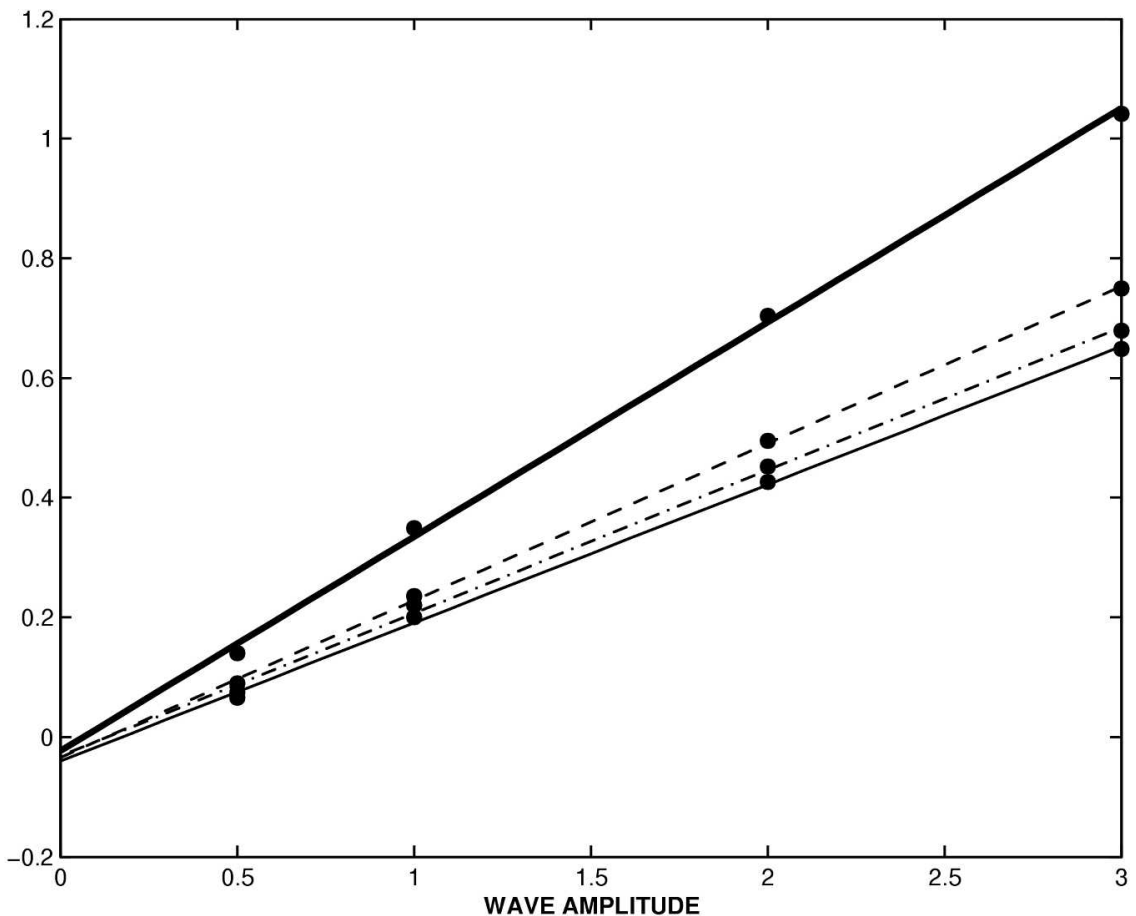

FIG. 8. Energy growth rate of the leading Floquet modes for $\mu=0$ (thick solid line), $\mu=$ 0.1 (dashed line), $\mu=0.2$ (dashed-dotted line), and $\mu=0.3$ (thin solid line) as a function of neutral wave amplitude. Filled circles denote the computed values, and lines show a least squares linear fit to these values. 

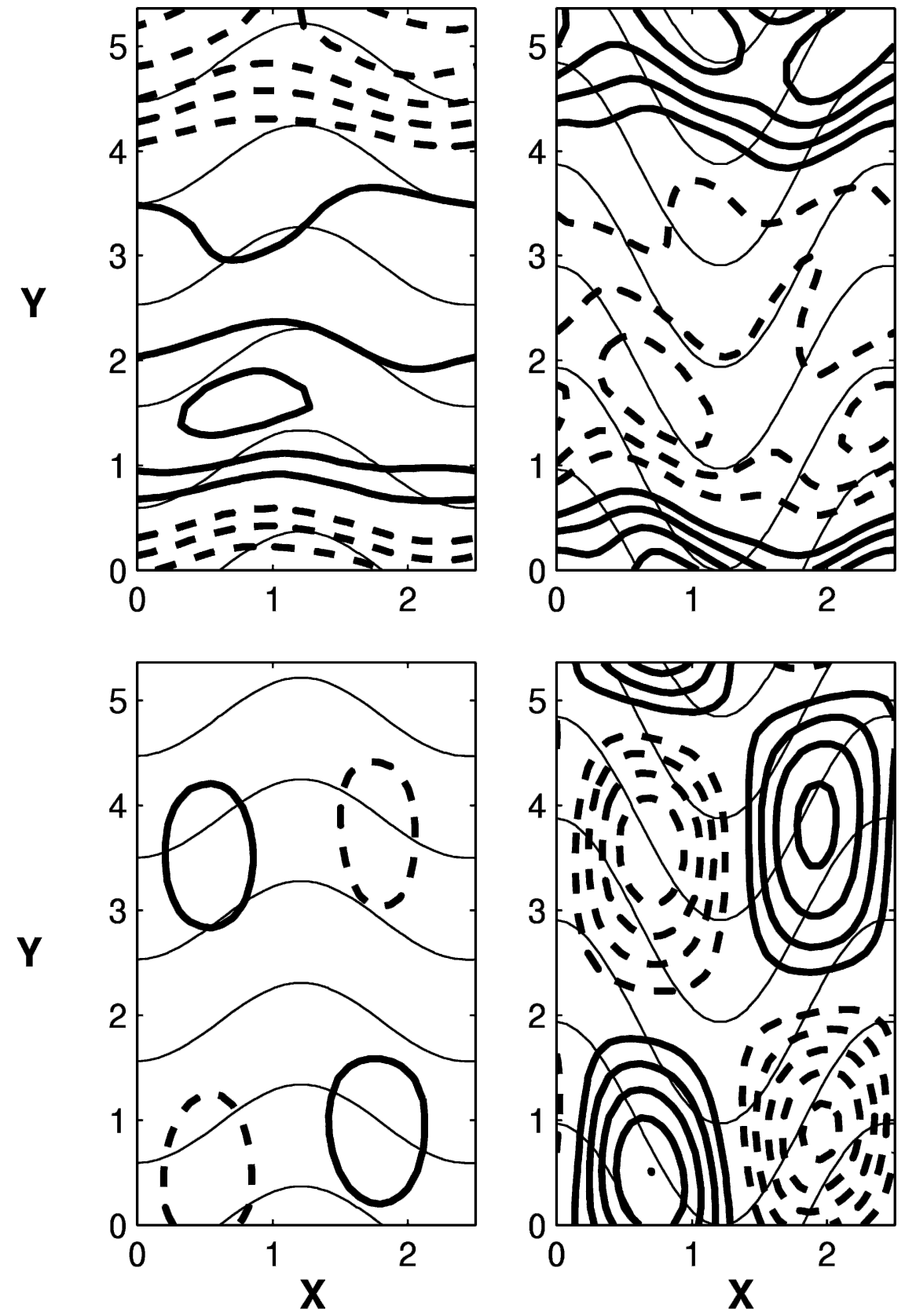

FIG. 9. Potential temperature at the (left) surface and (right) tropopause for the leading potential-enstrophy singular vector of the $\mu=0$ jet with a neutral wave amplitude of 1 . Thin solid lines show the basic state every 1 unit, and thick lines show the singular vector (top) every 0.2 units for the initial time and (bottom) every 0.8 units at $t=4.371$ time units; negative values are dashed.

fined by the leading eigenvector of $\mathbf{L}^{\mathrm{H}}+\mathbf{L}$ (e.g., Nolan and Farrell 1999). Optimal modes are particularly useful because they eliminate the ambiguity of optimization time in the singular vector calculation. The leading singular vector for the aperiodic basic state is similar to the singular vectors shown in Fig. 9, although with more banded structure in $y$ (not shown). The fastest growing instantaneous optimal mode also displays similarities to the leading Floquet mode, which suggests that this structure is important for growth not only over long time periods, but also at any instant (Fig. 11; cf. Fig. 2). 


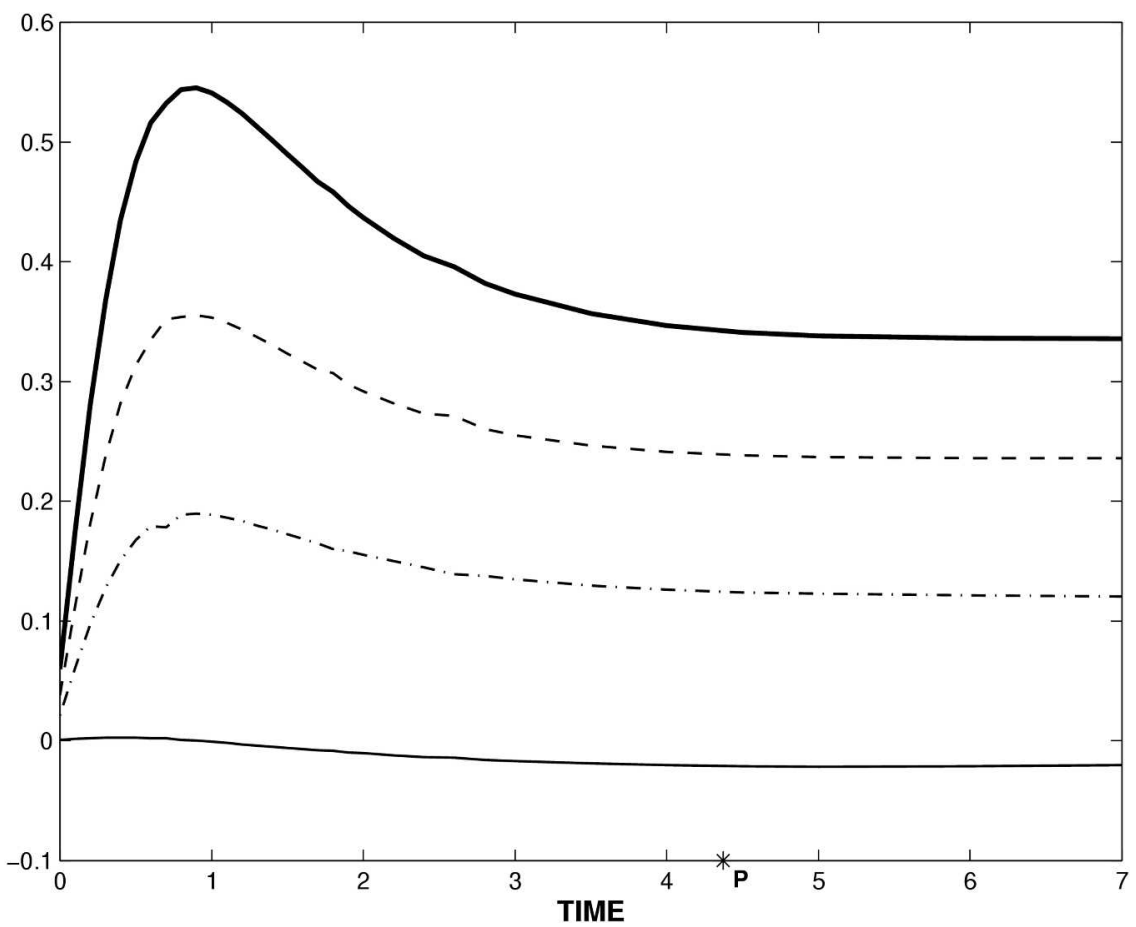

FIG. 10. Energy growth rate (thick solid line), barotropic term (dashed line), zonal heat flux term (dashed-dotted line), and meridional heat flux term (thin solid line) as a function of time for the potential enstrophy singular vector shown in Fig. 9. The asterisk labeled "P" denotes one basic-state period, which is also the singular vector optimization time.

\section{Initial-value problems}

Initial-value problems are solved in the quasigeostrophic spectral model described in section $3 \mathrm{~b}$ to determine the evolution of a localized initial condition. Specifically, the initial condition consists of a localized Gaussian superposed on a jet plus a neutral mode with a wavelength of $2500 \mathrm{~km}$. To allow the disturbance ample room to spread, the length of the domain is extended in the $x$ direction to $50(50000 \mathrm{~km})$, and a barotropic wind is added to the basic state to render the neutral wave stationary.

When the amplitude of the basic-state neutral wave is smaller than about $1(9.18 \mathrm{~K})$, the solution is dominated by the leading mode for the unperturbed jet (not shown); solutions for amplitudes above this threshold are dominated by the leading Floquet mode. For example, a solution after eight time units $(\approx 74 \mathrm{~h})$ for a neutral wave amplitude of $3(27.5 \mathrm{~K})$ clearly shows the emergence of the leading Floquet mode in the potential temperature field (Fig. 12). An $x-t$ diagram illustrates the evolution for this case at $y=1.383$ (Fig. 13), which corresponds to the location of the maximum Floquetmode amplitude. A wavelike pattern can be seen spreading both upstream and downstream from the initial disturbance. The wind speed at the tropopause level in this case is $0.4281\left(\approx 12.84 \mathrm{~m} \mathrm{~s}^{-1}\right)$ and localized disturbances growing in Eady shear cannot spread downstream faster than this (e.g., Farrell 1983). Apparently, adding a neutral wave to the Eady jet enables disturbances to spread upstream and downstream much faster than the mean flow. The phase speed of the Eady Floquet mode is estimated to be 0.42 in this coordinate system and the minimum and maximum group speeds, represented by the heavy dashed lines, are estimated to be -2.59 and 2.32 , respectively. In the absence of the barotropic wind the phase speed is $0.99\left(29.98 \mathrm{~m} \mathrm{~s}^{-1}\right)$, nearly equivalent to the tropopause zonal wind (30 $\left.\mathrm{m} \mathrm{s}^{-1}\right)$, and the group speeds are $-2.02\left(-60.54 \mathrm{~m} \mathrm{~s}^{-1}\right)$ and $2.89\left(86.70 \mathrm{~m} \mathrm{~s}^{-1}\right)$.

The initial-value problem is repeated for the $\mu=$ 0.2 Floquet problem. At $t=8(\approx 74 \mathrm{~h})$ the disturbance has not yet completely evolved to the leading Floquet mode; however, aspects of the Floquet-mode structure are apparent in the potential temperature field (Fig. 14). Again, the perturbations have small amplitude in the center of the domain, so we examine the $x-t$ development along $y=1.383$. A wavelike structure spreads with time both upstream and downstream from the initial disturbance (Fig. 15). As was the case for the Eady Floquet initial-value problem, the disturbance spreads much faster than the mean flow, which is indi- 

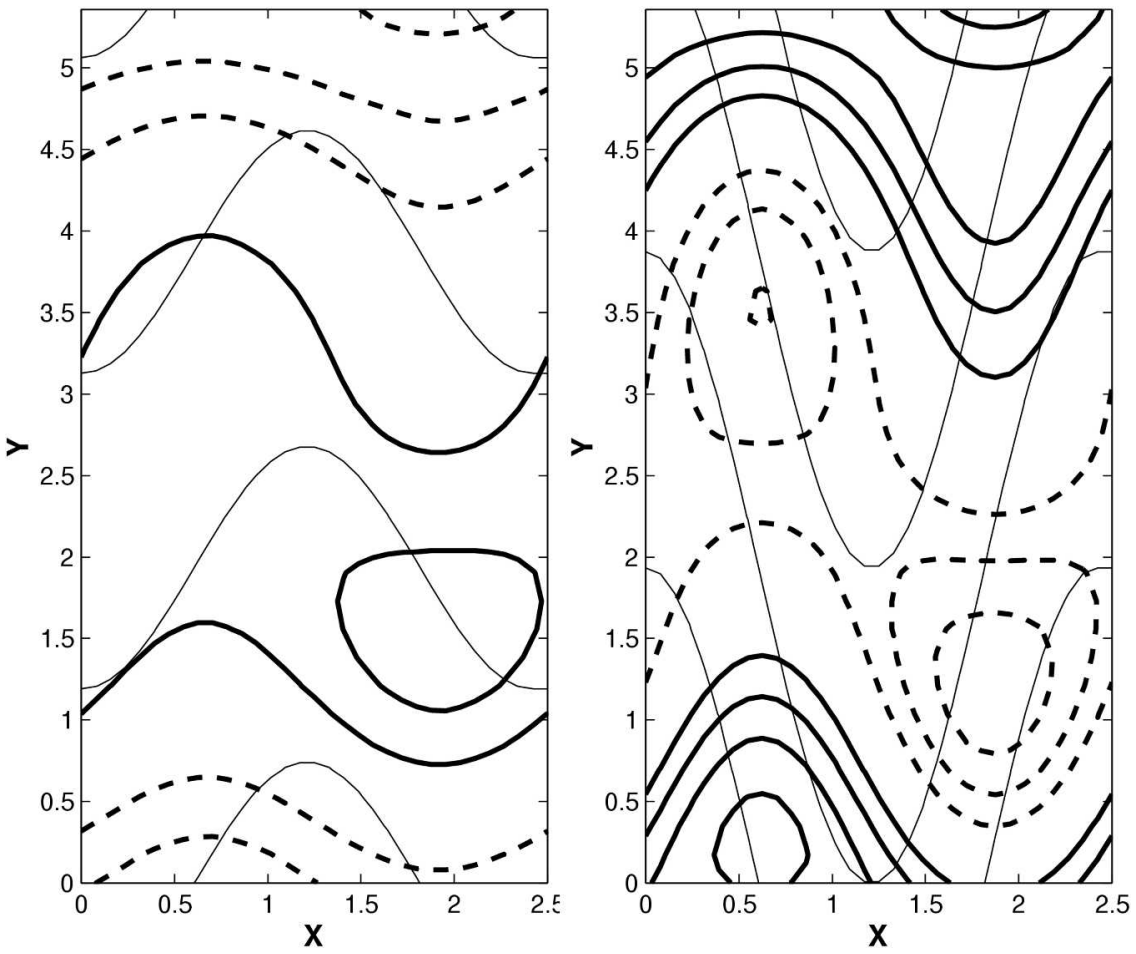

FIG. 11. Potential temperature contours every 0.4 units for the leading instantaneous optimal mode of the $\mu=0$ jet with a neutral wave of amplitude 2 at the (left) surface and (right) tropopause. The basic-state potential temperature is given every two units.

cated by the estimated group speeds of -2.62 and 1.27 , which are equivalent to $-2.12\left(-63.67 \mathrm{~m} \mathrm{~s}^{-1}\right)$ and 1.77 (53.03 $\mathrm{m} \mathrm{s}^{-1}$ ) without the barotropic wind. The phase speed of the leading mode is estimated at 0.34 in this coordinate system, or $0.84\left(25.13 \mathrm{~m} \mathrm{~s}^{-1}\right)$ in the absence of the barotropic wind, compared to a jet wind speed of $0.88\left(26.42 \mathrm{~m} \mathrm{~s}^{-1}\right)$.

\section{Discussion}

Floquet theory is applied to a periodic basic state consisting of a neutral wave and a baroclinic jet. The neutral wave adds horizontal shear and baroclinicity in the zonal direction, and destabilizes the jet to wavelengths that would otherwise be stable. Unstable modes for this flow, Floquet vectors, have growth rates that increase linearly with neutral wave amplitude and exhibit no threshold amplitude for the onset of instability. These modes have phase speeds similar to tropopauselevel wind speeds, and group speeds that exceed the jet-level winds.

Above a critical tropopause potential temperature amplitude of about 8-10 K, the growth rate of the Flo-

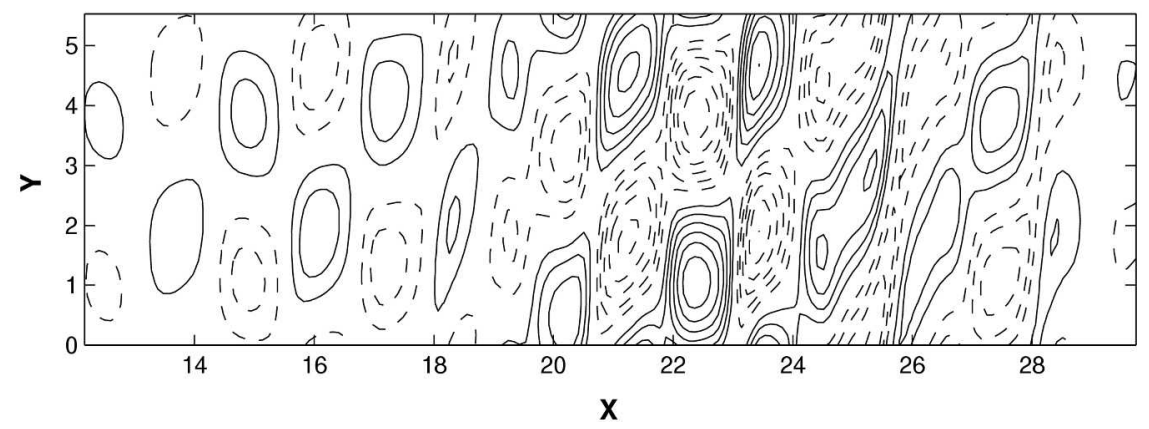

FIG. 12. Disturbance tropopause potential temperature after eight time units for a locally perturbed $\mu=0$ jet with a neutral wave of amplitude 3 . The initial condition consists of a tropopause-level Gaussian warm disturbance at the center of the domain $(x=25, y=2.77)$. Note that only a portion of the full computational domain is depicted in order to focus on the region of dominant activity. 


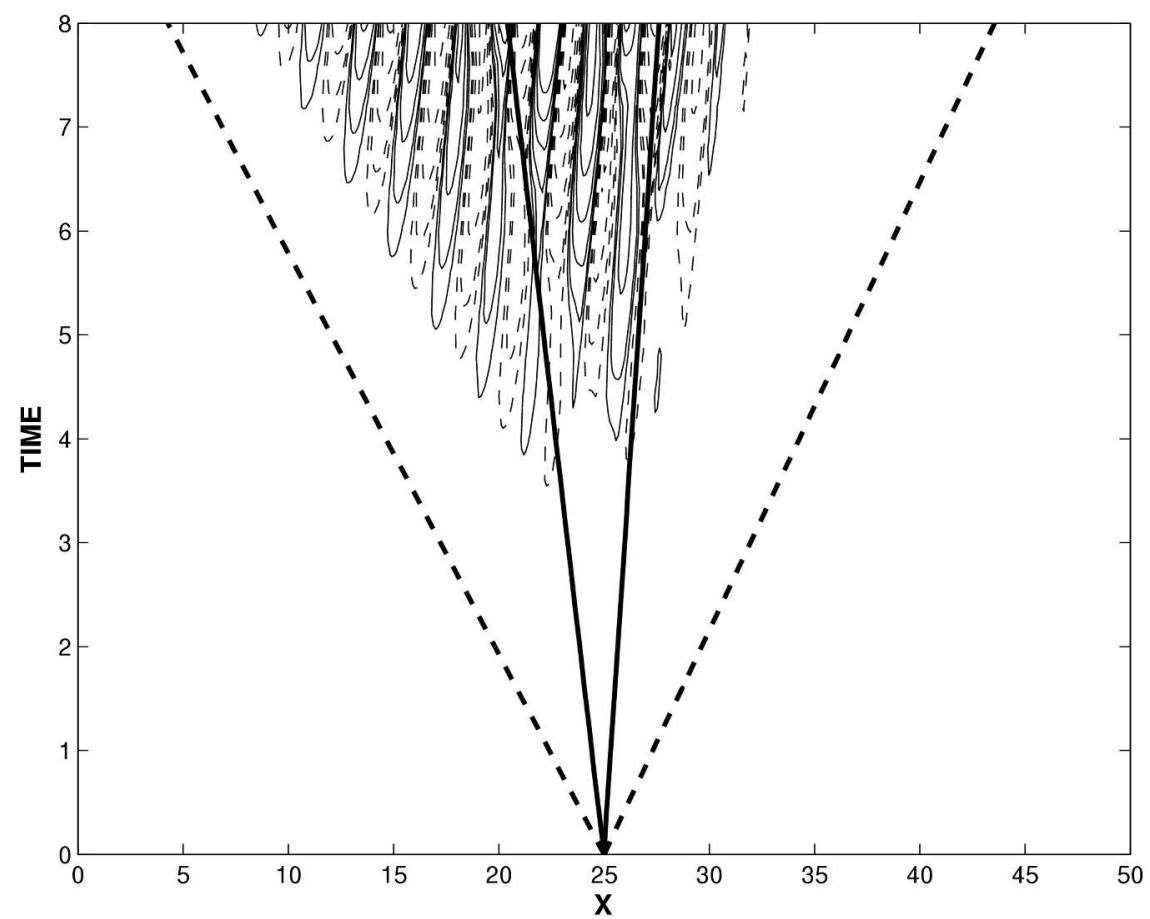

FIG. 13. The $x-t$ evolution of tropopause potential temperature at $y=1.383$ for a locally perturbed $\mu=0$ jet with a neutral wave amplitude of 3 . Contours are $\pm[0.1,0.2,0.4,0.7,1.1$, 1.6] units; negative values dashed. Thick solid lines show $x_{0}+\bar{u}_{s} t$ and $x_{0}+\bar{u}_{t} t$, where $\bar{u}_{s}$ and $\bar{u}_{t}$ represent the basic-state wind at the surface and tropopause, respectively. The thick dashed lines are the estimated group velocities.

quet mode exceeds that for the modes for the unperturbed, plane-parallel jet. A transition occurs at this amplitude, above which a localized initial condition evolves into the leading Floquet mode. Furthermore, the localized disturbance spreads spatially faster than the zonal flow both upstream and downstream. Thus, unlike the zonal jet, the jet with superposed baroclinic waves exhibits absolute instability, so that a fixed observer sees exponential growth for all time (e.g., Huerre and Monkewitz 1990).

The Floquet modes examined here are distinctly different from the normal modes of past studies of baro- clinic instability for jets with horizontal shear. For example, James (1987) and Nakamura (1993) studied the effects of horizontal shear on baroclinic instability and found a negative contribution from barotropic energy conversion. In contrast, barotropic conversion is responsible for about $70 \%$ of the Floquet-mode growth and the zonal heat flux is responsible for the remaining $30 \%$; the meridional heat flux does not contribute to the growth of the Floquet modes. Barotropic growth has also been shown to be important for the growth of frontal instabilities. For example, Joly and Thorpe (1990) assessed the stability of a two-dimensional front

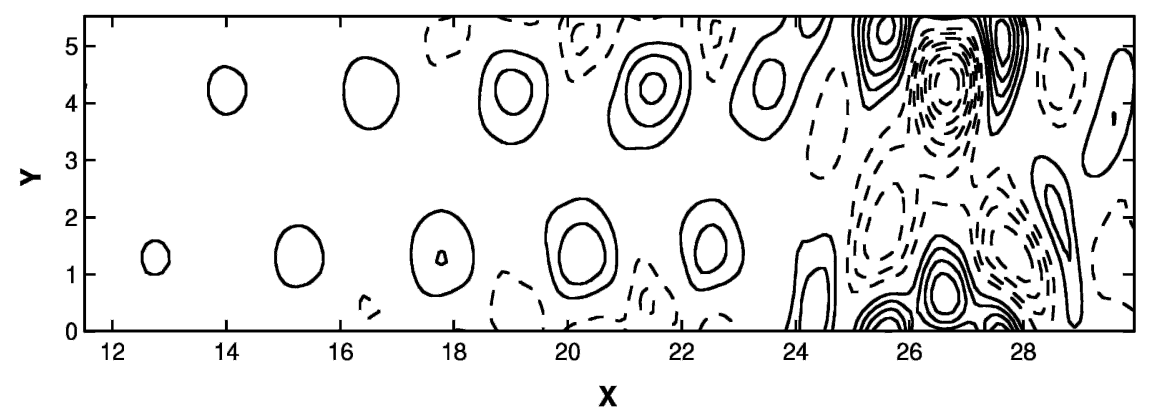

FIG. 14. As in Fig. 12, except for $\mu=0.2$ with a contour interval of 0.05 units. 


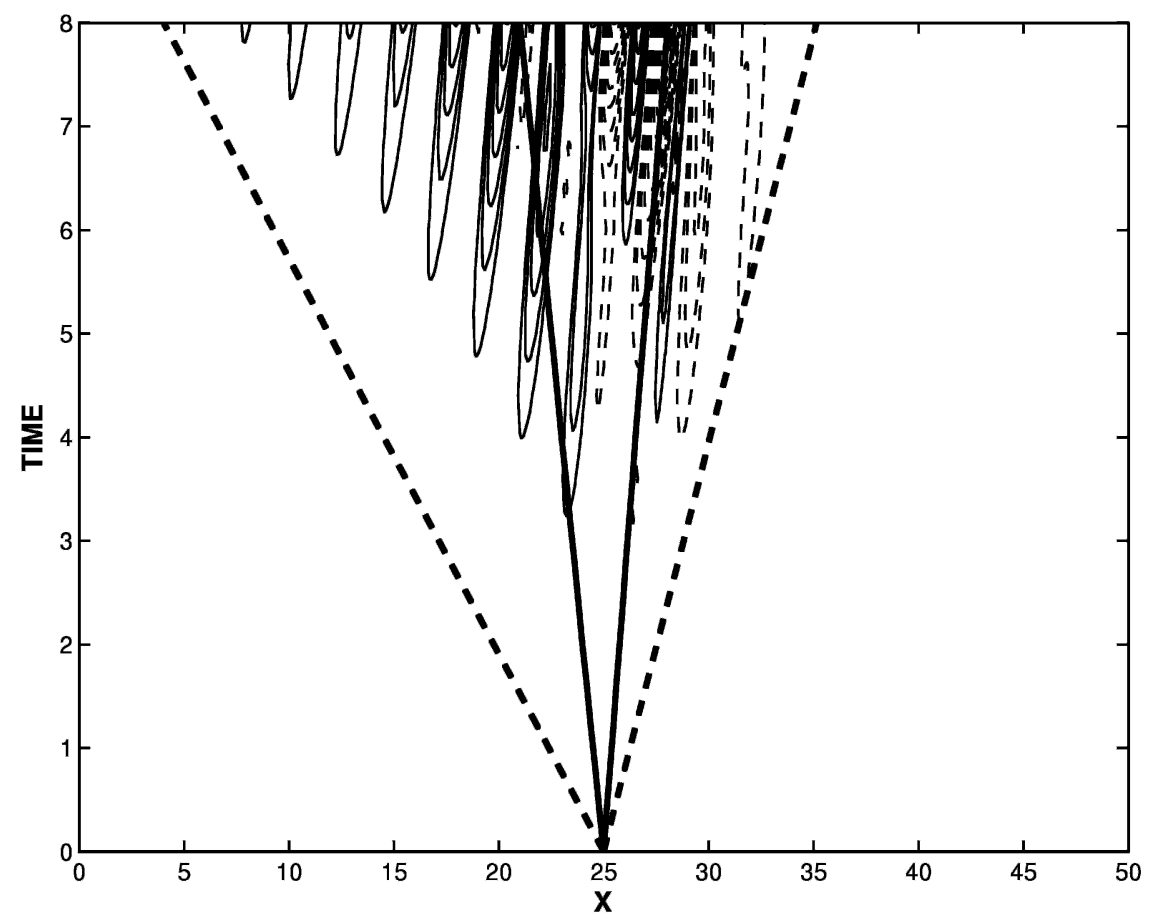

FIG. 15. As in Fig. 13, except for $\mu=0.2$ and contours of $\pm[0.04,0.08,0.12,0.16,0.20]$ units.

and found the primary source of growth was from the baroclinic terms; however, barotropic conversion also contributed significantly. Furthermore, the leadingmode growth rate was found to be a linear function of the Eady wave amplitude, as is the case here.

Our leading Floquet mode for the Eady jet recovers the second class of unstable modes of Fantini and Davolio (2001). These authors also studied periodic jets of the Eady type, and found that the growth rates exceed those for unstable modes of the Eady jet when the neutral wave amplitudes are greater than about 10-12 $\mathrm{hPa}(8-10 \mathrm{~K})$. Unlike this earlier study, the present study resolves all growing modes; for the Eady problem that Fantini and Davolio considered, there is a second growing mode with a growth rate comparable to the leading mode. We also find Floquet modes for small neutral wave amplitude; in fact, we find no evidence for a threshold amplitude for instability.

The structure of the Floquet modes changes smoothly with neutral wave wavelength, even beyond the short-wave cutoff; however, the growth mechanisms change slightly when crossing the short-wave cutoff since the basic-state wave in this case has vertical tilt. Barotropic conversion remains the leading term in the energetics budget, but the meridional heat flux becomes positive and contributes significantly. Undamped, aperiodic, growing waves also support growing modes similar to the leading Floquet modes for neutral wave basic states, implying that our results may have relevance to more general aperiodic flows.

Singular vectors, although not a main focus of this paper, were found for the Euclidean norm in boundary potential temperature over one basic-state period. The leading singular vector converges to the leading Floquet mode during the period. Furthermore, the leading instantaneous optimal mode has structure similar to the leading Floquet mode. In a related calculation, Snyder and Joly (1998) examined the singular vectors for a growing baroclinic wave on the HW jet using a streamfunction variance norm. Singular vectors for this flow initially have largest amplitude at the edges of the jet, similar to our concentrated-jet Floquet modes. Furthermore, barotropic conversion dominates the growth of Snyder and Joly's optimal modes, as was the case here.

As alluded to earlier, the stability analyses presented here may be useful for understanding the development of forecast errors in a storm track. For example, as the jet becomes more meridionally localized, the leading Floquet mode becomes concentrated off the jet axis, which implies the potential for greater forecast-error growth at the edges of the jets. The fact that a localized perturbation, such as an analysis or forecast error, spreads faster than the jet-level flow above a certain baroclinic wave amplitude presents a testable hypothesis for real forecast-error data. Preliminary circumstantial evidence that this hypothesis may be worth ex- 
ploring derives from the recent study of Hakim (2005), who found that analysis errors and short-term forecast errors in the Pacific storm track peak at the tropopause and have a dominant wavelength of about $3000 \mathrm{~km}$. Moreover, for zonal wavenumber 9 at $40^{\circ} \mathrm{N}$, analysis errors spread downstream at the speed of the zonal wind, although the data were not stratified by baroclinic wave amplitude. Assessing any relationship between these observational results and the Floquet solutions shown here is a subject for future research.

Acknowledgments. We thank three anonymous reviewers for their comments, which helped clarify aspects of the paper, and Dave Muraki for discussions on Floquet theory. This research was sponsored by the National Science Foundation through Grants ATM0228804 and CMG-0327658, and by the National Oceanic and Atmospheric Administration through CSTAR Grant NA17RJ1232. MRS was sponsored by an NSF Graduate Fellowship, and this study represents a portion of her master's thesis research at the University of Washington.

\section{REFERENCES}

Abramowitz, M., and I. A. Stegun, 1972: Handbook of Mathematical Functions with Formulas, Graphs, and Mathematical Tables. U.S. Government Printing Office, 1046 pp.

Benjamin, T. N., and F. Ursell, 1954: The stability of the plane free surface of a liquid in vertical periodic motion. Proc. Roy. Soc. London, A225, 505-515.

Drazin, P. G., and W. H. Reid, 1981: Hydrodynamic Stability. Cambridge University Press, 527 pp.

Duffy, D. G., 1978: The stability of a nonlinear, finite-amplitude neutrally stable Eady wave. J. Atmos. Sci., 35, 1619-1625.

Eady, E. T., 1949: Long waves and cyclone waves. Tellus, 1, 33-52.

Fantini, M., and S. Davolio, 2001: Instability of neutral Eady waves and orography. J. Atmos. Sci., 58, 1146-1154.

Farrell, B. F., 1983: Pulse asymptotics of three-dimensional baroclinic waves. J. Atmos. Sci., 40, 2202-2209.

_ 1989: Optimal excitation of baroclinic waves. J. Atmos. Sci., 46, 1193-1206.

— II: Nonautonomous operators. J. Atmos. Sci., 53, 2041-2053.

Hakim, G. J., 2000: Role of nonmodal growth and nonlinearity in cyclogenesis initial-value problems. J. Atmos. Sci., 57, 29512967.

, 2003: Developing wave packets in the North Pacific storm track. Mon. Wea. Rev., 131, 2824-2837.
- 2005: Vertical structure of midlatitude analysis and forecast errors. Mon. Wea. Rev., 133, 567-578.

Harnik, N., and R. S. Lindzen, 1998: The effect of basic-state potential vorticity gradients on the growth of baroclinic waves and the height of the tropopause. J. Atmos. Sci., 55, 344-360.

Hart, J. E., 1971: A note on the baroclinic instability of general time-dependent basic fields of the Eady type. J. Atmos. Sci., 28, 808-809.

Hoskins, B. J., and N. V. West, 1979: Baroclinic waves and frontogenesis. Part II: Uniform potential vorticity flows-Cold and warm fronts. J. Atmos. Sci., 36, 1663-1680.

Huerre, P., and P. A. Monkewitz, 1990: Local and global instabilities in spatially developing flows. Annu. Rev. Fluid Mech., 22, 473-537.

James, I. N., 1987: Suppression of baroclinic instability in horizontally sheared flows. J. Atmos. Sci., 44, 3710-3720.

Joly, A., and A. J. Thorpe, 1990: The stability of a steady horizontal shear front with uniform potential vorticity. J. Atmos. Sci., 47, 2612-2622.

Legras, B., and R. Vautard, 1995: A guide to Lyapunov vectors. Proc. ECMWF Seminar on Predictability, Vol. 1, Reading, United Kingdom, ECMWF, 143-156.

Lim, G. H., and J. M. Wallace, 1991: Structure and evolution of baroclinic waves as inferred from regression analysis. $J$. Atmos. Sci., 48, 1718-1732.

Molteni, F., and T. N. Palmer, 1993: Predictability and finite-time instability of the northern winter circulation. Quart. J. Roy. Meteor. Soc., 119, 269-298.

Nakamura, N., 1993: Momentum flux, flow symmetry, and the nonlinear barotropic governor. J. Atmos. Sci., 50, 2159-2179.

Nolan, D. S., and B. F. Farrell, 1999: Generalized stability analyses of asymmetric disturbances in one- and two-celled vortices maintained by radial inflow. J. Atmos. Sci., 56, 1287-1307.

Pedlosky, J., 1987: Geophysical Fluid Dynamics. 2d ed. SpringerVerlag, $710 \mathrm{pp}$.

Rotunno, R., and M. Fantini, 1989: Petterssen's "type B" cyclogenesis in terms of discrete, neutral Eady modes. J. Atmos. Sci., 46, 3599-3604.

Samelson, R. M., 2001: Periodic orbits and disturbance growth for baroclinic waves. J. Atmos. Sci., 58, 436-450.

_ , and C. L. Wolfe, 2003: A nonlinear baroclinic wave-mean oscillation with multiple normal mode instabilities. J. Atmos. Sci., 60, 1186-1199.

Snyder, C., and A. Joly, 1998: Development of perturbations within growing baroclinic waves. Quart. J. Roy. Meteor. Soc., 124, 1961-1983.

Song, Y., and N. Nakamura, 2000: Eady instability of isolated baroclinic jets with meridionally varying tropopause height. $J$. Atmos. Sci., 57, 46-65.

Yakubovich, V. A., and V. M. Starzhinskii, 1975: Linear Differential Equations with Periodic Coefficients. John Wiley and Sons, 386 pp. 